\title{
Response of Soil Microbial Community Structure and Function to Different Altitudes in Arid Valley in Panzhihua, China
}

Runji Zhang

Sichuan Agricultural University

Xianrui Tian

Sichuan Agricultural University

Quanju Xiang

Sichuan Agricultural University

Petri Penttinen

Sichuan Agricultural University

Yunfu Gu ( $\nabla$ guyf@sicau.edu.cn )

Sichuan Agricultural University

\section{Research Article}

Keywords: Arid valley, Altitudinal gradients, Soil properties, Microbial communities, Functional prediction

Posted Date: November 29th, 2021

DOI: https://doi.org/10.21203/rs.3.rs-1055315/v1

License: (c) (i) This work is licensed under a Creative Commons Attribution 4.0 International License. Read Full License

Version of Record: A version of this preprint was published at BMC Microbiology on April 2nd, 2022. See the published version at https://doi.org/10.1186/s12866-022-02500-6. 
1 Response of soil microbial community structure and function to different altitudes in arid valley in

2 Panzhihua, China

3 Runji Zhang ${ }^{\dagger}$, Xianrui Tian ${ }^{\dagger}$, Quanju Xiang, Petri Penttinen, Yunfu Gu*

4 Department of Microbiology, College of Resources, Sichuan Agricultural University, Chengdu

5 611130, China

6

7

8

9

10

11

12

13

14

15

$16 \uparrow$ Equal contributor

$17{ }^{*}$ Corresponding author

18 E-mail: guyf@sicau.edu.cn 


\section{Abstract}

Background: Altitude affects biodiversity and physic-chemical properties of soil, providing natural sites for studying species distribution and the response of biota to environmental changes. We sampled soil at three altitudes in an arid valley, determined the physic-chemical characteristics and microbial community composition in the soils, identified differentially abundant taxa and the relationships between community composition and environmental factors.

Results: The low, medium and high altitudes were roughly separated based on the physic-chemical characteristics and clearly separated based on the microbial community composition. The differences in community composition were associated with differences in all measured factors except $\mathrm{pH}$. The contents of organic and microbial biomass $\mathrm{C}$, total and available $\mathrm{N}$ and available $\mathrm{P}$, and the richness and diversity of the microbial communities were lowest in the medium altitude. The relative abundances of phyla Proteobacteria, Gemmatimonadetes, Actinobacteria and Acidobacteria were high at all altitudes. The differentially abundant ASVs were mostly assigned to Proteobacteria and Acidobacteria. The highest number of ASVs characterizing altitude were detected in the high altitude. However, the predicted functions of the communities were overlapping, suggesting that the contribution of the communities to soil processes changed relatively little along the altitude gradient.

Conclusions: The composition of microbial community at different altitudes was related to the differences of all measuring factors except $\mathrm{pH}$ in arid valley in Panzhihua, China.

Keywords: Arid valley; Altitudinal gradients; Soil properties; Microbial communities; Functional prediction 


\section{Background}

Altitude affects biodiversity and physic-chemical properties of soil; the plant cover, soil properties and climate change dramatically with altitude in mountain ecosystems, providing natural sites for studying species distribution and the response of biota to environmental changes [1]. Knowledge on altitude related biodiversity patterns is important for understanding the impacts of climate change on ecosystems [2].

Microbial communities represent the most abundant form of life in the soil, playing an important role in $\mathrm{C}$ and nutrient cycles. The communities respond rapidly to and are affected by environmental changes $[3,4]$. The activity and structure of microbial communities are affected by soil type, temperature, vegetation and other abiotic and biological factors [5]. To date, the research on microbial communities in mountain ecosystems has mostly focused on plateaus and frozen soils $[6,7]$. The results on microbial diversity along altitude gradients have been contradictory; diversity has been found to decrease with altitude, to be highest at mid-altitude, and to show no clear patterns of change [1, 8-10]. In Changbai Mountain in China, the composition of bacterial communities changed along the altitude gradient $[11,12]$. The changes in community composition and microbial biomass and activity were accompanied with changes in vegetation type and soil physic-chemical properties, e.g $\mathrm{C}$ and $\mathrm{N}$ content and $\mathrm{pH}$ [13-15].

Panzhihua in the south of Sichuan, China, at the interchange between Jinsha River and Yalong River, has a subtropical climate with abundant rainfall and a hot rainy season. However, high evapotranspiration due to strong sunshine and valley winds result in an arid local climate in the arid valley areas $[16,17]$. Soil in Panzhihua is typically acid red soil, and the area of soil with $\mathrm{pH}$ lower than 5.5 accounts for almost $16 \%$ of the total soil area [18]. Information on the effects of 
altitude and environmental factors on soil microbial communities in arid valleys is still limited.

Since the temperature and air humidity in this region vary considerably within a small range of altitude difference, we expected that the soil microbial community structure would vary accordingly. In this study, we sampled soil at three altitudes in an arid valley, determined the physic-chemical characteristics and microbial community composition in the soils, identified differentially abundant taxa and the relationships between community composition and environmental factors, providing a basis for the understanding of soil ecosystems in arid valleys.

\section{Results}

\section{Soil physic-chemical characteristics}

The soil $\mathrm{pH}$ and temperature were lowest and $\mathrm{AK}$ content was highest at the high altitude (2000 m a.s.l.) $(\mathrm{P}<0.05)($ Table 1). Soil moisture was lowest and TK content was highest at the low altitude $(1600 \mathrm{~m}$ a.s.l. $)(\mathrm{P}<0.05)$. TP content increased with increasing altitude $(\mathrm{P}<0.05)$. SOC, TN, AP, AN, and SMBC contents were highest at the high altitude and lowest at the medium altitude (1800 m a.s.l.) $(\mathrm{P}<0.05)$. SMBN content was lowest at the medium altitude. Based on the physicochemical properties, the soil samples were roughly separated according to the altitude (Fig. S1).

\section{Microbial communities}

The richness of the microbial communities was highest at the low altitude and lowest at the medium altitude $(\mathrm{P}<0.05)$ (Table 2). The diversity was lowest at the medium altitude $(\mathrm{P}<0.05)$. The richness of the microbial communities correlated negatively with soil moisture and the diversity correlated positively with $\mathrm{TN}$, AN and SMBN $(\mathrm{P}<0.05)$ (Table 3). The relative abundances of phyla Proteobacteria were above $40 \%$, and those of Gemmatimonadetes and 
Actinobacteria were above $12 \%$ at all altitudes (Fig.2A). The relative abundances of genera Sphingomonas, Gemmatimonas and Saccharimonadales were high at all altitudes (Fig. 2B). Based on the Bray-Curtis dissimilarities between samples, the communities grouped according to the altitude in the non-metric multidimensional scaling (NMDS) (Fig. 3A). The analysis of similarities (ANOSIM) showed that the microbial communities at different altitudes were different $(\mathrm{P}<0.001$; Table S1). Distance-based redundancy analysis (dbRDA) showed that the differences in community composition were associated with differences in all soil properties except $\mathrm{pH}(\mathrm{P}<0.05)$

(Fig. 3B). Specifically, the difference between communities at high and low altitudes was associated with differences in soil temperature and TK and AK contents, the difference between communities at high and medium altitudes was associated with differences in SOC, TN and AN content, and the difference between communities at medium and low altitudes was associated with a difference in SMBN content.

Out of the 58802 ASVs, the 3674 ASVs with an observed mean abundance higher than one were included in the differential abundance analysis. Altogether 354 differentially abundant ASVs were detected between the low and medium altitudes, 384 between the low and high altitudes, and 408 between the medium and high altitudes (Table S2). ASVs differentially abundant in comparing one altitude to both the other altitudes were taken as characteristic to the altitude. Ten and three ASVs were enriched and depleted, respectively, in the low altitude compared to both the medium and high altitude (Table 4). The relative abundance of the ten and three ASVs characteristic to low altitude decreased and increased, respectively, constantly along the altitude. One enriched ASV was characteristic to the medium altitude (Table 4). Altogether 152 enriched and 32 ASVs were characteristic to the high altitude (Table 4). Most of the altitude characteristic 
ASVs were assigned to phyla Proteobacteria and Acidobacteria, and at lower taxonomic levels to uncultured and unclassified taxa (Table 4). Out of the well-defined taxa, Anaeromyxobacter, Gemmatimonas, Sphingomonas, Bryobacter, Flavisolibacter and Phenylobacterium included both enriched and depleted ASVs (Table 4).

\section{S rRNA gene based functional prediction}

Based on the predicted functions, the communities were separated according to the altitude, yet the separation was not as clear as that based on the taxonomic community composition PCoA showed that the microbial communities might harbor distinct ecological functions at different altitudes (Fig. 4A). Similarly, the differences in functional composition were associated with differences in all soil properties except $\mathrm{pH}(\mathrm{P}<0.05)$ (Fig. 4B).

\section{Discussion}

The diversity and activity of microbial communities depend on temperature and other climatic variables that change substantially along altitude $[1,2]$. Thus, altitude gradients provide natural sites to assess the effects of environmental change on the communities. To date, the diversity, composition and function of microbial communities along altitude gradients under various climates have received attention $[5,19,20]$. To estimate whether the communities in arid climate vary similarly, we studied microbial community composition and the relationships between community composition and environmental factors along an altitude gradient in an arid valley in Panzhihua, China.

With the increase of altitude, the change in climate and vegetation result in changes in soil physic-chemical properties $[13,21,22]$. Below tree line, the SOC and TN contents increased with the increase of altitude $[13,23]$. In our study, the low and high-altitude sites in the arid valley were 
clearly different based on the physical properties. The available $\mathrm{K}$ and available $\mathrm{P}$ contents were over two to over seven times higher in the high than in the lower altitudes. Out of the measured chemical properties, the contents of organic and microbial biomass $\mathrm{C}$, total and available $\mathrm{N}$ and available P were different at different altitudes; however, the contents were lowest in the medium altitude and highest in the high altitude. Similarly, the richness and diversity of the microbial communities were lowest in the medium altitude. Accumulation of SOC at the high-altitude site was possibly due to the lower temperature that is known to slow down soil respiration [24]. The availability of $\mathrm{N}$ and $\mathrm{P}$ decrease below $\mathrm{pH} 6$ and 4.5, respectively, thus the differences in $\mathrm{pH}$ between sites were not expected to affect the availability of $\mathrm{P}$ and have only a minor effect on the availability of $\mathrm{N}$. Possibly the availability of $\mathrm{N}$ and $\mathrm{P}$ were governed by differences in plant uptake of the nutrients and in $\mathrm{N}$ and $\mathrm{P}$ cycle related microorganisms. As the total $\mathrm{P}$ content increased with the increasing altitude, the differences in the $\mathrm{P}$ contents may have been due to differences in bedrock along the altitude gradient.

The relative abundances of phyla Proteobacteria, Gemmatimonadetes, Actinobacteria and Acidobacteria, all among the OTU richest phyla on earth [25], were high at all altitudes. Similar to earlier studies $[1,26]$, the microbial community compositions at the altitudes were distinct. The differentially abundant ASVs were mostly assigned to Proteobacteria and Acidobacteria. The highest number of ASVs characterizing altitude were detected in the high altitude, suggesting a bigger difference between the high and lower altitudes than that revealed by community composition only. In contrast to the community composition, the predicted functions of the communities were overlapping. The sharing of functions by taxonomically distinct communities, i.e. functional redundancy [27], suggested that the contribution of the communities to soil 
processes, e.g. nutrient and $\mathrm{C}$ cycling, changed relatively little along the altitude gradient.

In assessing the relationships between the microbial community composition and environmental factors, the differences in community composition were associated with differences in all measured factors except $\mathrm{pH}$. On a continental scale over a wide $\mathrm{pH}$ range, soil $\mathrm{pH}$ was the master variable in explaining variation in microbial communities [28]. Presumably the effect of $\mathrm{pH}$ on the communities is less pronounced over a relatively narrow $\mathrm{pH}$ range as in our study. Specifically, the difference between communities at high and low altitudes was associated with differences in soil temperature and TK and AK contents, the difference between communities at high and medium altitudes was associated with differences in SOC, TN and AN content, and the difference between communities at medium and low altitudes was associated with a difference in

\section{SMBN content.}

\section{Conclusion}

The low, medium and high altitudes were roughly separated based on the physicochemical characteristics and clearly separated based on the microbial community composition. The differences in community composition were associated with differences in all measured factors except $\mathrm{pH}$. The overlapping functions suggested that the contribution of the communities to soil processes changed relatively little along the altitude gradient.

\section{Methods}

\section{Study area and soil sampling}

Anning valley in Miyi, Panzhihua, Sichuan, China (E102 ${ }^{\circ} 7^{\prime}, \mathrm{N}^{2} 6^{\circ} 76^{\prime}, 1587-2108 \mathrm{~m}$ a.s.l) has a typical subtropical valley climate. The mean annual temperature ranges from 15 to $29{ }^{\circ} \mathrm{C}$, with maximum and minimum temperatures of $34{ }^{\circ} \mathrm{C}$ in May and $13{ }^{\circ} \mathrm{C}$ in January, respectively. 
172

173

174

175

176

177

178

179

180

181

Annual precipitation is approximately 800 to $1200 \mathrm{~mm}$.

Fifteen $20 \times 20 \mathrm{~m}$ sampling plots with at least $100 \mathrm{~m}$ distance between the plots were established in July 28, 2018, at 1600, 1800 and 2000 m a.s.l (Fig. 1). Within each plot, five topsoil $(0-10 \mathrm{~cm})$ subsamples were taken from the center and each corner using a 5 -cm diameter soil corer after removing litter from the soil surface by hand. The subsamples were combined into one composite soil sample per plot. The composite samples were divided into two portions: the portion for physic-chemical analyses was stored at $4{ }^{\circ} \mathrm{C}$ and the portion for DNA extraction was stored at $-80{ }^{\circ} \mathrm{C}$.

\section{Soil physic-chemical properties}

The soil samples were air-dried, ground and sieved through a 2-mm mesh. Soil pH was measured in a soil-to-water ratio of 1:1 using a glass electrode $\mathrm{pH}$ meter (FiveGo, Mettler Toledo, Greifensee, Switzerland). Soil organic carbon (SOC) and total nitrogen (TN) contents were determined using dichromate oxidization and Kjeldahl digestion [29], and available nitrogen (AN) as described earlier [30]. Total phosphorus (TP) and total potassium (TK) contents were determined after digestion in $\mathrm{HF}_{-} \mathrm{HClO}_{4}$. Soil moisture (MO) content was measured by drying the fresh soil samples at $105^{\circ} \mathrm{C}$ until a constant weight [31]. Available phosphorus (AP) and available potassium $(\mathrm{AK})$ contents were determined using sodium bicarbonate and ammonium acetate extraction [13, 32]. Soil microbial biomass C (SMBC) and biomass N (SMBN) contents were estimated by using chloroform fumigation extraction as described earlier [33].

\section{DNA extraction, amplification and sequencing}

DNA was extracted from 1.21-1.45 g fresh weight soil (corresponding to approximately 0.50 g dry weight) using the Fast DNA Spin Kit for Soil (MP Bio medicals, Solon, OH, USA) 
194

195

following the manufacturer's instructions. Concentration and quality of the extracted DNA were examined using Nano-200 spectrophotometer (Aosheng, Hangzhou, China) and agars gel electrophoresis. DNA extracts were stored at $-20{ }^{\circ} \mathrm{C}$ until further processing. The V4 hyper variable region of $16 \mathrm{~S}$ rRNA gene was amplified using primers $515 \mathrm{~F}$ (5'-GTGCC-AGCMGCCGCGGTAA-3') and 806R (5'-GGACTACVSGGGTATCTAAT-3') with adapter and barcode sequences [34]. Amplification was done in a $50 \mu \mathrm{L}$ reaction mixture with $3 \mathrm{U}$ of TaKaRa Ex Taq HS (TaKARA Shuzo Co., Shiga, Japan), 5 mM dNTP mixture (TaKARA Shuzo Co., Shiga, Japan), $2.0 \mathrm{mM} \mathrm{MgCl} 2,5 \mu \mathrm{L}$ of 10× Ex Taq Buffer (TaKARA Shuzo Co., Shiga, Japan), $0.6 \mathrm{mM}$ of each primer, and $4.0 \mathrm{ng}$ of DNA. The amplification program in an S1000 thermo cycler (Bio-Rad Laboratories, CA, USA) included an initial denaturation at $94{ }^{\circ} \mathrm{C}$ for 4 min, and 30 cycles of $15 \mathrm{~s}$ at $94{ }^{\circ} \mathrm{C}, 15 \mathrm{~s}$ at $55^{\circ} \mathrm{C}$ and $30 \mathrm{~s}$ at $72{ }^{\circ} \mathrm{C}$, and a final extension at $72{ }^{\circ} \mathrm{C}$ for $10 \mathrm{~min}$.

The PCR products from three replicate amplifications per sample were pooled and purified with AxyPrep DNA Purification Kit (Axygen Biotech, Hangzhou, China), and quantified using PR omega QuantiFluor (Invitrogen, Carlsbad, CA, USA). Purified amplicons were pooled in equimolar concentrations and sequenced using MiSeq Reagent Kit V3 on an Illumina MiSeq platform (Illumina Biotech, California, USA) at Shanghai Personal Biotechnology Co. Ltd, Shanghai, China. The sequence data were submitted to NCBI Sequence Read Archive (https://www.ncbi.nlm.nih.gov/sra/) with accession number PRJNA663774.

\section{Bioinformatics analysis}

The sequence data were processed and assigned into amplicon sequence variants (ASVs) using R package DADA2 [35]. The ASVs were assigned to taxa using Silva release 132 database 

QIIME software (Version 1.7.0).

\section{Statistical analysis}

Differences were regarded as statistically significant at $p<0.05$. Relative abundances at phylum and genus levels were visualized as percentages using the multtest package in $\mathrm{R}$ package vegan version 2.4.4 in $\mathrm{R}$ v.3.3.2. [36]. Differences in the soil properties and microbial alpha diversities were tested with two-way ANOVA using the Statistical Package for the Social Sciences (SPSS Version 19.0, SPSS Inc., Chicago, IL, USA). The associations between microbial alpha diversity and environmental factors were analyzed using Pearson correlation [37]. The heterogeneity of the variance was tested, and the original data were normalized using log-transformation or standardization prior to analysis when necessary. Non-metric multidimensional scaling (NMDS) and distance based redundancy analysis (dbRDA) were carried out using the Vegan package in R v.3.4 [38]. Differentially abundant ASVs were determined in pairwise comparisons using Aldex2[39]. ASVs with observed mean abundance $<1$ were excluded from the analysis. ASVs with an expected value of the Benjamini-Hochberg corrected t-test $\mathrm{p}<$ $0.05 / 3$ (the number of comparisons) were taken as differentially abundant. The functions of the communities were predicted using phylogenetic investigation of communities by reconstruction of unobserved states (PICRUSt) (Langille et al., 2013). 


\section{Declarations}

\section{Ethics approval and consent to participate}

All experiments on microbial were conducted in accordance with the cultivars protection

239 regulations of the Sichuan Agricultural University.

\section{Consent for publication}

241 Not applicable.

\section{Availability of data and materials}

243 The sequence data were submitted to NCBI Sequence Read Archive

244 (https://www.ncbi.nlm.nih.gov/sra/) with accession number PRJNA663774. The datasets used 245 or analyzed during the current study are available from the corresponding author on 246 reasonable request.

\section{Competing interests}

248 The authors declare that they have no competing interests.

\section{$249 \quad$ Funding}

The study was funded by the National Natural Science Foundation of China (grant No. 41201256) and Key Research and Development Program of the Department of Science and Technology of Sichuan province (grant No. 2020YFN0100).

\section{Authors' contributions}

254 Runji Zhang and Yunfu Gu designed the experiments. Runji Zhang performed the 255 experiments and completed the analysis of soil physic-chemical properties, Xianrui Tian completed field sampling and data analysis. Quanju Xiang performed the bioinformatics and 
manuscript. All authors approved the final version of the manuscript.

\section{Acknowledgments}

260 We thanks to Yan Xiao et al. for participating in the experiment, and thanks to Yingyan Wang and

261 Ming Liu for help in data analysis. 
263 1. Ren C, Zhang W, Zhong Z, Han X, Yang G, Feng Y, Ren G: Differential responses of soil microbial biomass, diversity, and compositions to altitudinal gradients depend on plant and soil characteristics. Sci Total Environ 2018, 610-611:750-758.

2. Donhauser J, Frey B: Alpine soil microbial ecology in a changing world. FEMS Microbiol Ecol 2018, 94(9).

3. Cavicchioli R, Ripple WJ, Timmis KN, Azam F, Bakken LR, Baylis M, Behrenfeld MJ, Boetius A, Boyd PW, Classen AT et al: Scientists' warning to humanity: microorganisms and climate change. Nature Reviews Microbiology 2019, 17(9):569-586.

4. Lin Y, Ye Y, Hu Y, Shi H: The variation in microbial community structure under different heavy metal contamination levels in paddy soils. Ecotoxicol Environ Saf 2019, 180:557-564.

5. Chang EH, Chen TH, Tian G, Chiu CY: The effect of altitudinal gradient on soil microbial community activity and structure in moso bamboo plantations. Applied Soil Ecology 2016, 98:213-220.

6. Delgado-Baquerizo M, Maestre FT, Reich PB, Trivedi P, Singh BK: Carbon content and climate variability drive global soil bacterial diversity patterns. Ecol Monogr 2016, 86(3).

7. Guo G, Kong W, Liu J, Zhao J, Du H, Zhang X, Xia P: Diversity and distribution of autotrophic microbial community along environmental gradients in grassland soils on the Tibetan Plateau. Appl Microbiol Biotechnol 2015, 99(20):8765-8776.

8. Singh D, Takahashi K, Kim M, Chun J, Adams JM: A Hump-Backed Trend in Bacterial Diversity with Elevation on Mount Fuji, Japan. Microb Ecol 2012, 63(2):429-437.

9. Wang JT, Cao P, Hu HW, Li J, Han LL, Zhang LM, Zheng YM, He JZ: Altitudinal distribution patterns of soil bacterial and archaeal communities along $\mathrm{mt}$. Shegyla on the Tibetan Plateau. Microb Ecol 2015, 69(1):135-145.

elevational diversity patterns of plants and animals. Ecology 2011, 92(4):797-804.

11. Liu D, Wu X, Shi S, Liu H, Liu G: A hollow bacterial diversity pattern with elevation in Wolong Nature Reserve, Western Sichuan Plateau. J Soils Sed 2016, 16(10):2365-2374.

12. Shen $\mathrm{C}$, Ni Y, Liang $\mathrm{W}$, Wang J, Chu H: Distinct soil bacterial communities along a small-scale elevational gradient in alpine tundra. Front Microbiol 2015, 6:582.

13. Hu L, Xiang Z, Wang G, Rafique R, Liu W, Wang C: Changes in soil physicochemical and microbial properties along elevation gradients in two forest soils. Scand J For Res 2016, 31(3):242-253.

14. Shen C, Shi Y, Fan K, He JS, Adams JM, Ge Y, Chu H: Soil pH dominates elevational diversity pattern for bacteria in high elevation alkaline soils on the Tibetan Plateau. FEMS Microbiol Ecol 2019, 95(2).

15. Wang J, Zhang T, Li L, Li J, Feng Y, Lu Q: The Patterns and Drivers of Bacterial and Fungal $\beta$-Diversity in a Typical Dryland Ecosystem of Northwest China. Frontiers in Microbiology 2017, 8:2126-.

Pei J, Yang W, Cai Y, Yi Y, Li X: Relationship between Vegetation and Environment in an Arid-Hot Valley in Southwestern China. Sustainability 2018, 10(12).

17. Xu KW, Zou L, Penttinen P, Zeng X, Liu M, Zhao K, Chen C, Chen YX, Zhang X: Diversity 
and phylogeny of rhizobia associated with Desmodium spp. in Panxi, Sichuan, China. Syst Appl Microbiol 2016, 39(1):33-40.

18. $\mathrm{Li} \mathrm{XL}$, Penttinen $\mathrm{P}, \mathrm{Gu} \mathrm{YF}$, Zhang XP: Diversity of nifH gene in rhizosphere and non-rhizosphere soil of tobacco in Panzhihua, China. Annals of Microbiology 2012, 62(3):995-1001.

19. Jin H, Yang X, Liu R, Yan Z, Li X, Li X, Su A, Zhao Y, Qin B: Bacterial community structure associated with the rhizosphere soils and roots of Stellera chamaejasme $L$. along a Tibetan elevation gradient. Annals of Microbiology 2018, 68(5):273-286.

20. Zhang L, Wang A, Yang W, Xu Z, Wu F, Tan B, Liu Y, Chen L: Soil microbial abundance and community structure vary with altitude and season in the coniferous forests, China. J Soils Sed 2016, 17(9):2318-2328.

21. Siles JA, Cajthaml T, Filipová A, Minerbi S, Margesin R: Altitudinal, seasonal and interannual shifts in microbial communities and chemical composition of soil organic matter in Alpine forest soils. Soil Biol Biochem 2017, 112:1-13.

22. Lu SB, Xu Y, Fu XP, Xiao H, Ding W, Zhang YJ: Patterns and Drivers of Soil Respiration and Vegetation at Different Altitudes in Southern China. Appl Ecol Environ Res 2019, 17(2):3097-3106.

23. Zhang Q, Wang D, Gong M, Zhang L: Changes in Physicochemical Properties of Forest Soil Along Different Altitudes in Huoditang of Qinling Mountains. Journal of Soil and Water Conservation 2011.

24. Müller M, Oelmann Y, Schickhoff U, Böhner J, Scholten T: Himalayan treeline soil and foliar C:N:P stoichiometry indicate nutrient shortage with elevation. Geoderma 2017, 291:21-32.

25. Louca S, Mazel F, Doebeli M, Parfrey LW: A census-based estimate of Earth's bacterial and archaeal diversity. PLoS Biol 2019, 17(2):e3000106.

26. Bayranvand M, Akbarinia M, Salehi Jouzani G, Gharechahi J, Kooch Y, Baldrian P: Composition of soil bacterial and fungal communities in relation to vegetation composition and soil characteristics along an altitudinal gradient. FEMS Microbiol Ecol 2020, 97(1).

27. Louca S, Polz MF, Mazel F, Albright MBN, Huber JA, O'Connor MI, Ackermann M, Hahn AS, Srivastava DS, Crowe SA et al: Function and functional redundancy in microbial systems. Nat Ecol Evol 2018, 2(6):936-943.

28. Fierer N, Jackson RB: The diversity and biogeography of soil bacterial communities. Proceedings of the National Academy of Sciences of the United States of America 2006, 103: (3) 626-631(3).

29. Bangroo SA, Najar GR, Rasool A: Effect of altitude and aspect on soil organic carbon and nitrogen stocks in the Himalayan Mawer Forest Range. Catena 2017, 158:63-68.

30. Feng W, Yang F, Cen R, Liu J, Qu Z, Miao Q, Chen H: Effects of straw biochar application on soil temperature, available nitrogen and growth of corn. J Environ Manage 2021, 277:111331.

31. Wei G, Li M, Shi W, Tian R, Chang C, Wang Z, Wang N, Zhao G, Gao Z: Similar drivers but different effects lead to distinct ecological patterns of soil bacterial and archaeal communities. Soil Biol Biochem 2020, 144.

32. Borin S, Ventura S, Tambone F, Mapelli F, Schubotz F, Brusetti L: Rock weathering creates 
oases of life in a High Arctic desert. Environ Microbiol 2010.

33. Zhen Z, Liu H, Wang N, Guo L, Meng J, Ding N, Wu G, Jiang G: Effects of manure compost application on soil microbial community diversity and soil microenvironments in a temperate cropland in China. PLoS One 2014, 9(10):e108555.

34. Caporaso JG, Lauber CL, Walters WA, Berg-Lyons D, Huntley J, Fierer N, Owens SM, Betley $\mathrm{J}$, Fraser L, Bauer $\mathrm{M}$ et al: Ultra-high-throughput microbial community analysis on the Illumina HiSeq and MiSeq platforms. ISME J 2012, 6(8):1621-1624.

35. Callahan BJ, McMurdie PJ, Rosen MJ, Han AW, Johnson AJA, Holmes SP: DADA2: High-resolution sample inference from Illumina amplicon data. Nat Methods 2016, 13(7):581-583.

36. Pollard KS, Gilbert HN, Ge Y, Taylor S, Dudoit S: Multtest: resampling-based multiple hypothesis testing. $R$ package version 2.17.0. $R$ Development Core Team. 2010. R: A language and environment for statistical computing. Available 2015.

37. Lauber CL, Hamady M, Knight R, Fierer N: Pyrosequencing-Based Assessment of Soil pH as a Predictor of Soil Bacterial Community Structure at the Continental Scale. Appl Environ Microbiol 2009, 75(15):5111-5120.

38. Oksanen J, Kindt R, Pierre L, O'Hara B, Simpson G, Solymos P: Vegan: Community Ecology Package. R Package Version 2.4-4. http://CRAN.R-project.org/package =vegan. 2017.

39. Fernandes AD, Macklaim JM, Linn TG, Gregor R, Gloor GB, John P: ANOVA-Like Differential Expression (ALDEx) Analysis for Mixed Population RNA-Seq. Plos One 2013, 8(7):e67019. 


\section{Figure legends}

373 Fig. 1 Location of the arid valley in Panzhihua city and sampling sites. High, altitude 2000 m a.s.1.; medium, altitude $1800 \mathrm{~m}$ a.s.l.; low, altitude $1600 \mathrm{~m}$ a.s.l.

375 Fig. 2 Relative abundance of dominant soil microbial community in different altitudes: A: genus

376 level; B phylum level. Low: High, altitude 2000 m a.s.l.; medium, altitude 1800 m a.s.l.; low,

377 altitude $1600 \mathrm{~m}$ a.s.l.

378 Fig. 3 Non-metric multidimensional scaling (NMDS) plot based on the Bray-Curtis dissimilarity between samples (A); Distance-based redundancy analysis (dbRDA) to quantify the impacts of environmental parameters on microbial community composition (B). High, altitude 2000 m a.s.1.; medium, altitude $1800 \mathrm{~m}$ a.s.l.; low, altitude $1600 \mathrm{~m}$ a.s.l. SOC: Soil organic carbon, MO: Moisture, TN: Total nitrogen, TP: Total phosphorus, TK: Total potassium, AN: Available nitrogen, AP: Available phosphorus, AK: Available potassium, ST: Soil temperature, SMBC: Soil microbial biomass carbon, SMBN: soil microbial biomass nitrogen.

Fig. 4 Principal co-ordinates analysis (PCoA) (A); Distance-based redundancy analysis (dbRDA) (B) of predicted functional profiles, significance at * $\mathrm{p}<0.05$; $* * \mathrm{p}<0.01$; $* * * \mathrm{p}<0.001$. High, altitude $2000 \mathrm{~m}$ a.s.l.; medium, altitude $1800 \mathrm{~m}$ a.s.1.; low, altitude $1600 \mathrm{~m}$ a.s.l. SOC: Soil organic carbon, MO: Moisture, TN: Total nitrogen, TP: Total phosphorus, TK: Total potassium, AN: Available nitrogen, AP: Available phosphorus, AK: Available potassium, ST: Soil temperature, SMBC: Soil microbial biomass carbon, SMBN: soil microbial biomass nitrogen. 

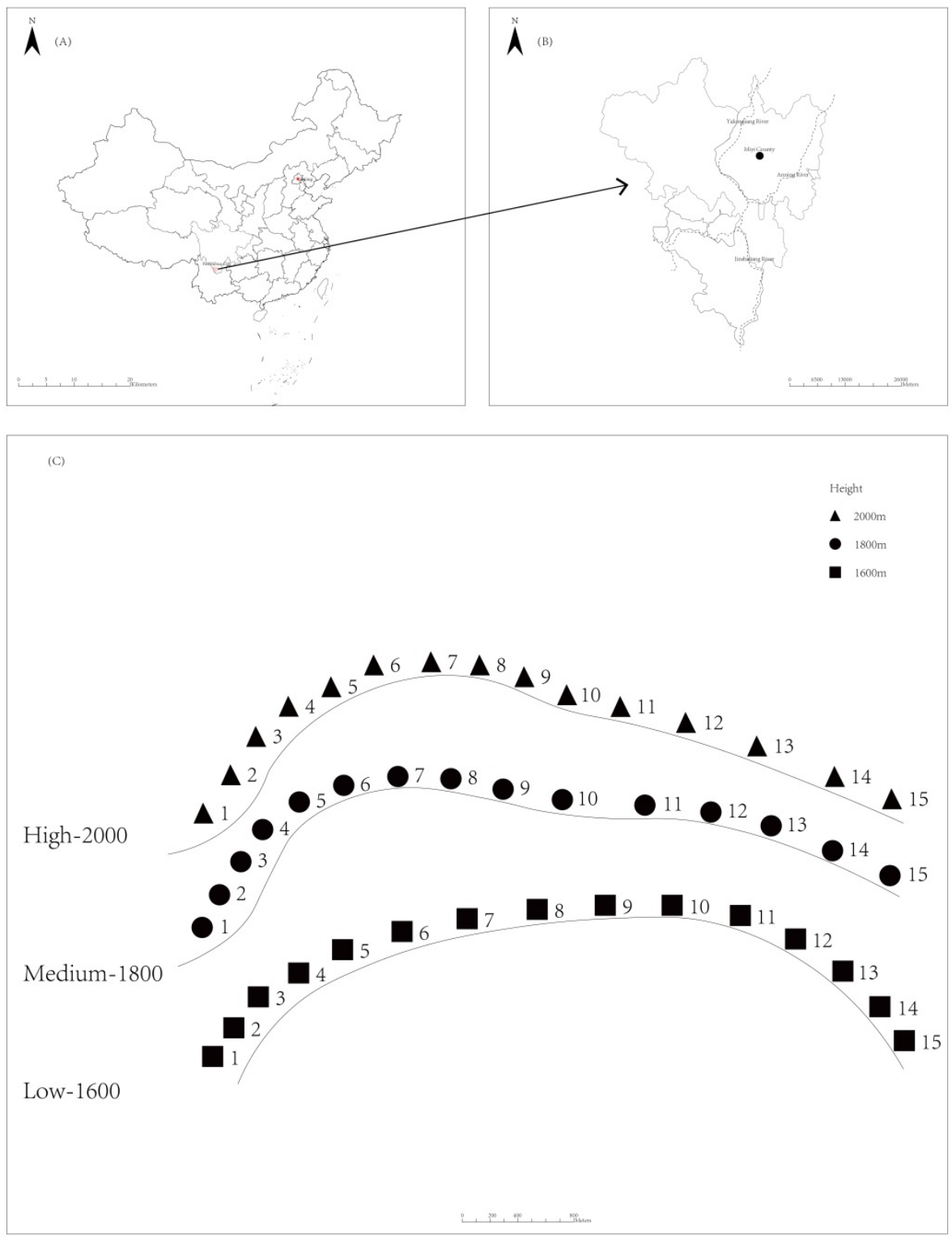

392 Fig. 1 Location of the arid valley and sampling sites in Panzhihua, China. High, altitude $2000 \mathrm{~m}$ 393 a.s.l.; medium, altitude $1800 \mathrm{~m}$ a.s.l.; low, altitude $1600 \mathrm{~m}$ a.s.l. 

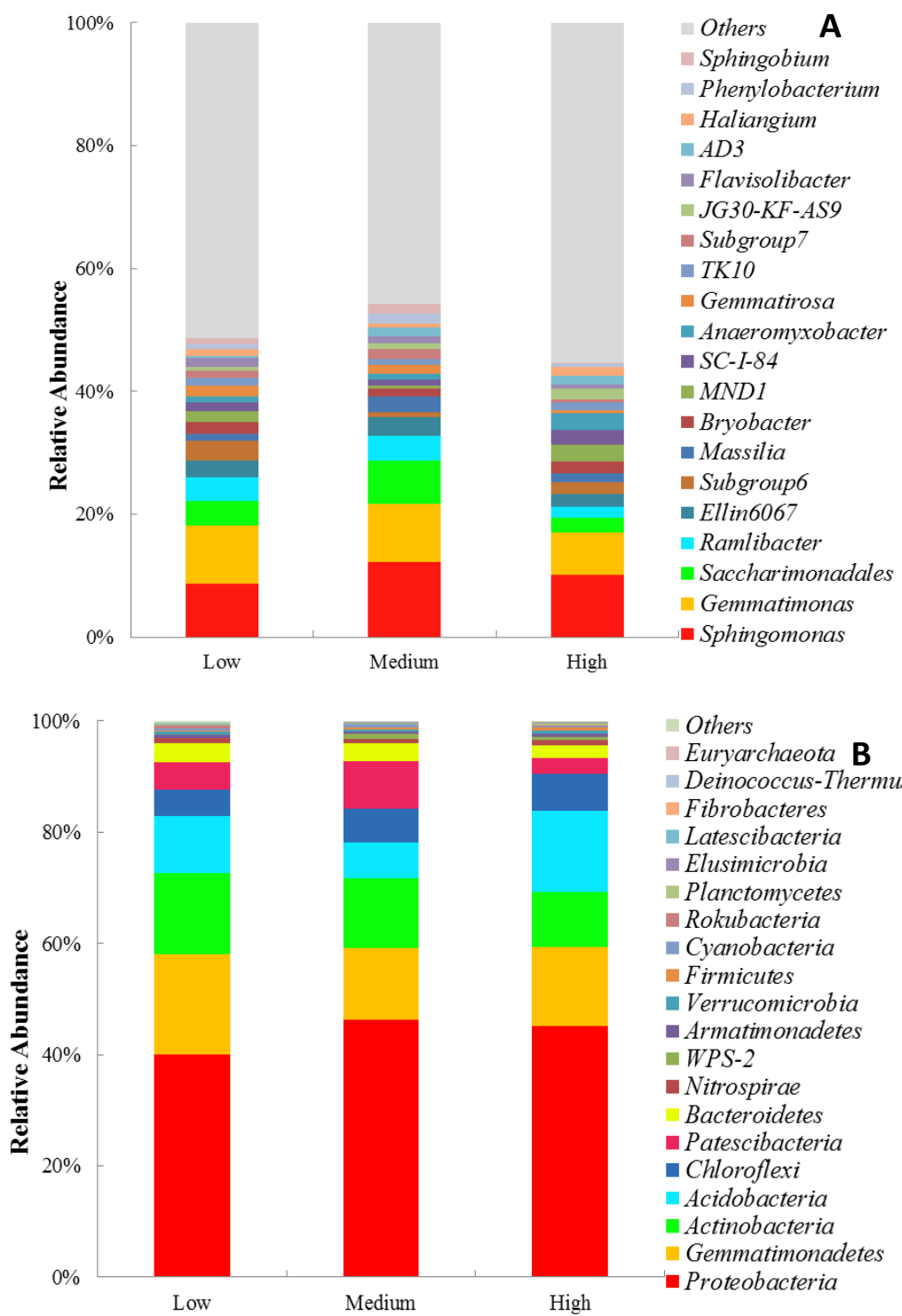

397 Fig. 2 Relative abundance of dominant soil microbial taxa in different elevations at A) genus level and B) phylum level. High, altitude $2000 \mathrm{~m}$ a.s.l.; medium, altitude $1800 \mathrm{~m}$ a.s.l.; low, altitude $1600 \mathrm{~m}$ a.s.1. 

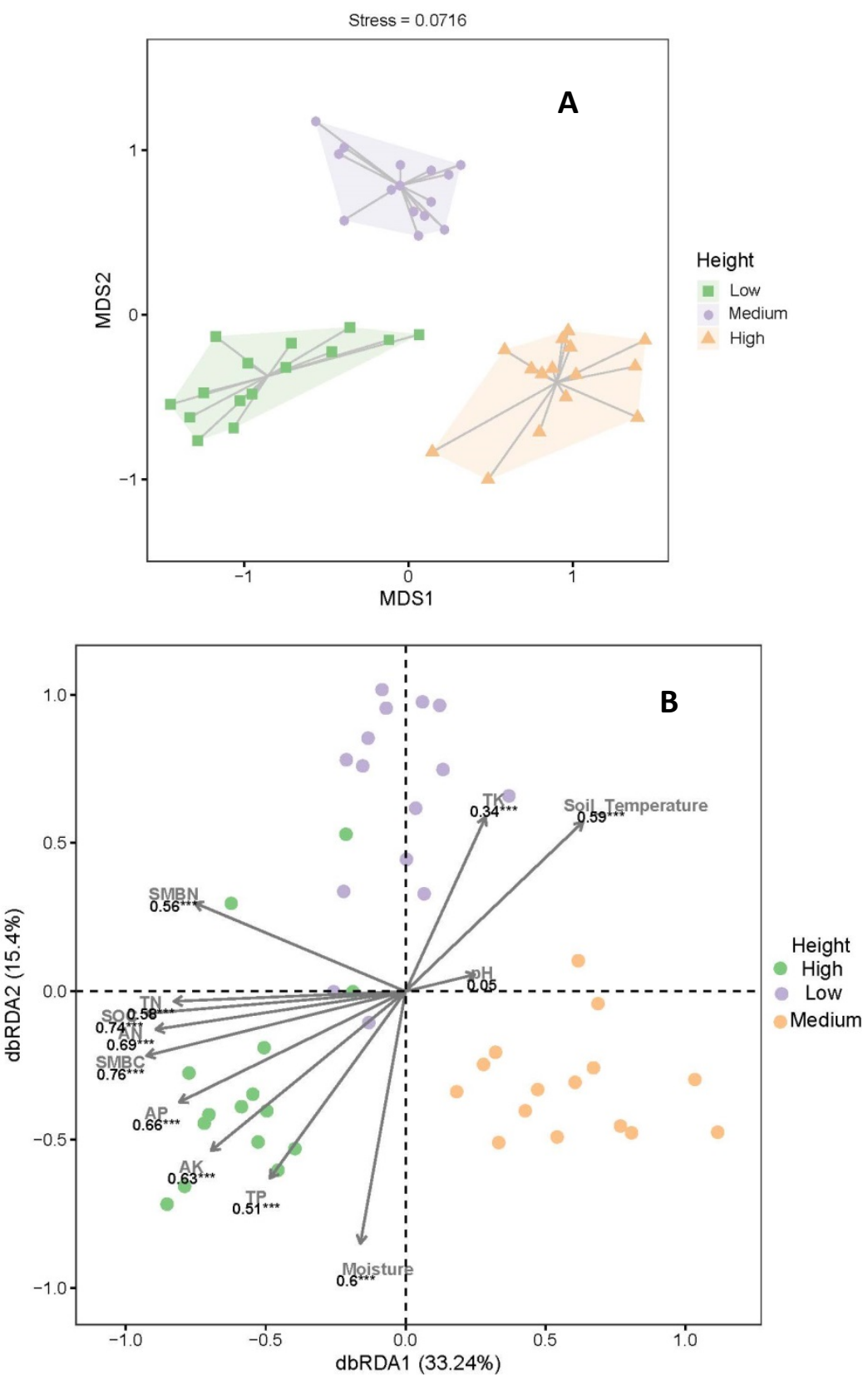

Fig. 3 Non-metric multidimensional scaling (NMDS) plot based on the Bray-Curtis dissimilarity between samples (A); Distance-based redundancy analysis (dbRDA) to quantify the impacts of environmental parameters on microbial community composition (B). High, altitude $2000 \mathrm{~m}$ a.s.l.; medium, altitude $1800 \mathrm{~m}$ a.s.l.; low, altitude $1600 \mathrm{~m}$ a.s.l. SOC: Soil organic carbon, MO: Moisture, TN: Total nitrogen, TP: Total phosphorus, TK: Total potassium, AN: Available nitrogen, AP: Available phosphorus, AK: Available potassium, ST: Soil temperature, SMBC: Soil microbial biomass carbon, SMBN: soil microbial biomass nitrogen. 

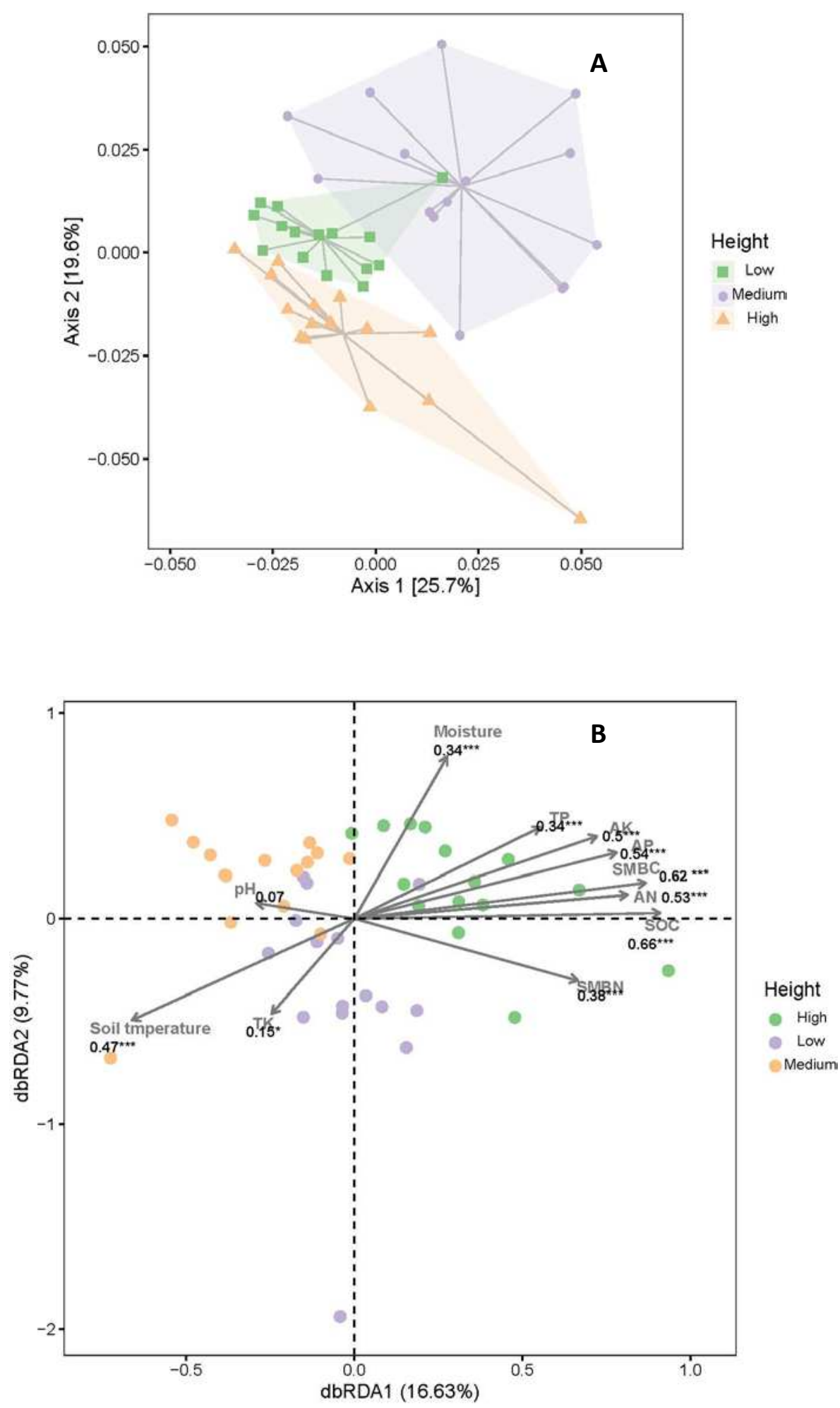

Fig. 4 Principal co-ordinates analysis (PCoA) of predicted functional profiles (A); Distance-based redundancy analysis (dbRDA) (B) of predicted functional profiles, significance at $* \mathrm{p}<0.05$; $* *$ $\mathrm{p}<0.01$; *** $\mathrm{p}<0.001$. High, altitude $2000 \mathrm{~m}$ a.s.l.; medium, altitude $1800 \mathrm{~m}$ a.s.l.; low, altitude $1600 \mathrm{~m}$ a.s.l.SOC: Soil organic carbon, MO: Moisture, TN: Total nitrogen, TP: Total phosphorus, TK: Total potassium, AN: Available nitrogen, AP: Available phosphorus, AK: Available potassium, ST: Soil temperature, SMBC: Soil microbial biomass carbon, SMBN: soil microbial biomass nitrogen. 
422 Table 1 The soil properties at different sites along the altitudes

\begin{tabular}{cccc}
\hline & Low & Medium & High \\
\hline $\mathrm{pH}$ & $5.45 \pm 0.42^{\mathrm{a}}$ & $5.40 \pm 0.26^{\mathrm{a}}$ & $5.17 \pm 0.13^{\mathrm{b}}$ \\
$\mathrm{SOC}\left(\mathrm{g} \cdot \mathrm{kg}^{-1}\right)$ & $10.20 \pm 1.02^{\mathrm{b}}$ & $6.30 \pm 0.67^{\mathrm{c}}$ & $16.38 \pm 1.34^{\mathrm{a}}$ \\
Moisture $(\%)$ & $27.25 \pm 1.54^{\mathrm{b}}$ & $32.09 \pm 1.39^{\mathrm{a}}$ & $33.31 \pm 1.91^{\mathrm{a}}$ \\
$\mathrm{TN}\left(\mathrm{g} \cdot \mathrm{kg}^{-1}\right)$ & $2.48 \pm 0.49^{\mathrm{b}}$ & $1.87 \pm 0.15^{\mathrm{c}}$ & $3.12 \pm 0.19^{\mathrm{a}}$ \\
$\mathrm{TP}\left(\mathrm{g} \cdot \mathrm{kg}^{-1}\right)$ & $1.07 \pm 0.49^{\mathrm{c}}$ & $1.16 \pm 0.10^{\mathrm{b}}$ & $1.33 \pm 0.06^{\mathrm{a}}$ \\
$\mathrm{TK}\left(\mathrm{g} \cdot \mathrm{kg}^{-1}\right)$ & $6.75 \pm 0.20^{\mathrm{a}}$ & $6.13 \pm 0.76^{\mathrm{b}}$ & $5.55 \pm 0.48^{\mathrm{b}}$ \\
$\mathrm{AN}\left(\mathrm{mg} \cdot \mathrm{kg}^{-1}\right)$ & $31.64 \pm 7.43^{\mathrm{b}}$ & $21.81 \pm 3.43^{\mathrm{c}}$ & $46.26 \pm 2.53^{\mathrm{a}}$ \\
$\mathrm{AP}\left(\mathrm{mg} \cdot \mathrm{kg}^{-1}\right)$ & $7.41 \pm 2.73^{\mathrm{b}}$ & $4.61 \pm 2.70^{\mathrm{c}}$ & $33.77 \pm 7.50^{\mathrm{a}}$ \\
$\mathrm{AK}\left(\mathrm{mg} \cdot \mathrm{kg}^{-1}\right)$ & $327.63 \pm 78.82^{\mathrm{b}}$ & $412.87 \pm 257.69^{\mathrm{b}}$ & $1094.40 \pm 121.32^{\mathrm{a}}$ \\
Temperature $\left({ }^{\circ} \mathrm{C}\right)$ & $25.07 \pm 0.54^{\mathrm{a}}$ & $24.32 \pm 1.59^{\mathrm{a}}$ & $20.91 \pm 0.99^{\mathrm{b}}$ \\
$\mathrm{SMBC}\left(\mathrm{mg} \cdot \mathrm{kg}^{-1}\right)$ & $90.67 \pm 13.05^{\mathrm{b}}$ & $62.08 \pm 9.68^{\mathrm{c}}$ & $156.80 \pm 4.92^{\mathrm{a}}$ \\
SMBN $\left(\mathrm{mg} \cdot \mathrm{kg}^{-1}\right)$ & $5.24 \pm 1.26^{\mathrm{a}}$ & $3.37 \pm 0.63^{\mathrm{b}}$ & $5.35 \pm 0.61^{\mathrm{a}}$ \\
\hline
\end{tabular}

423 Notes: Data are average \pm SEM $(n=3)$. Different letters in a column denote statistically significant 424 differences $(\mathrm{p}<0.05)$. SOC: Soil organic carbon, MO: Moisture, TN: Total nitrogen, TP: Total 425 phosphorus, TK: Total potassium, AN: Available nitrogen, AP: Available phosphorus, AK: Available potassium, ST: Soil temperature, SMBC: Soil microbial biomass carbon, SMBN: soil microbial biomass nitrogen. High, altitude $2000 \mathrm{~m}$ a.s.l.; medium, altitude $1800 \mathrm{~m}$ a.s.l.; low, altitude $1600 \mathrm{~m}$ a.s.l. 
Table 2 Alpha diversity of bacterial diversity at different sites along the altitudes

\begin{tabular}{ccc} 
Altitude & Chao1 & Shannon \\
\hline High & $1970.45 \pm 306.98^{\mathrm{b}}$ & $0.996 \pm 0.001^{\mathrm{a}}$ \\
Medium & $1645.68 \pm 177.23^{\mathrm{c}}$ & $0.992 \pm 0.002^{\mathrm{b}}$ \\
Low & $2121.47 \pm 193.67^{\mathrm{a}}$ & $0.997 \pm 0.001^{\mathrm{a}}$ \\
\hline
\end{tabular}

430 Notes: Data are average \pm SEM. Different letters in a column denote statistically significant 431 differences ( $\mathrm{p}$ < 0.05). High, altitude $2000 \mathrm{~m}$ a.s.l.; medium, altitude $1800 \mathrm{~m}$ a.s.l.; low, altitude $4321600 \mathrm{~m}$ a.s.l. 
433 Table 3 Pearson correlation coefficient of Alpha diversity of bacterial diversity and environmental 434 factors

\begin{tabular}{ccc}
\hline & Chao1 & Shannon \\
\hline $\mathrm{pH}$ & 0.483 & -0.560 \\
$\mathrm{SOC}\left(\mathrm{g} \mathrm{kg}^{-1}\right)$ & 0.043 & 0.910 \\
Moisture (\%) & $-0.934^{*}$ & -0.106 \\
$\mathrm{TN}\left(\mathrm{g} \mathrm{kg}^{-1}\right)$ & 0.166 & $0.954^{*}$ \\
$\mathrm{TP}\left(\mathrm{g} \mathrm{kg}^{-1}\right)$ & -0.662 & 0.367 \\
$\mathrm{TK}\left(\mathrm{g} \mathrm{kg}^{-1}\right)$ & 0.777 & -0.208 \\
$\mathrm{AN}\left(\mathrm{mg} \mathrm{kg}^{-1}\right)$ & 0.061 & $0.917 *$ \\
$\mathrm{AP}\left(\mathrm{mg} \mathrm{kg}^{-1}\right)$ & -0.260 & 0.742 \\
$\mathrm{AK}\left(\mathrm{mg} \mathrm{kg}^{-1}\right)$ & -0.436 & 0.603 \\
$\mathrm{Temperature}\left({ }^{\circ} \mathrm{C}\right)$ & 0.496 & -0.548 \\
$\mathrm{SMBC}\left(\mathrm{mg} \mathrm{kg}^{-1}\right)$ & -0.051 & 0.866 \\
$\mathrm{SMBN}\left(\mathrm{mg} \mathrm{kg}^{-1}\right)$ & 0.605 & $0.984^{*}$ \\
\hline
\end{tabular}

435 Notes: $* \mathrm{P}<0.001$. High, altitude $2000 \mathrm{~m}$ a.s.l.; medium, altitude $1800 \mathrm{~m}$ a.s.l.; low, altitude 1600 436 m a.s.l. SOC: Soil organic carbon, MO: Moisture, TN: Total nitrogen, TP: Total phosphorus, TK: 437 Total potassium, AN: Available nitrogen, AP: Available phosphorus, AK: Available potassium, 438 ST: Soil temperature, SMBC: Soil microbial biomass carbon, SMBN: soil microbial biomass 439 nitrogen. High, altitude $2000 \mathrm{~m}$ a.s.l.; medium, altitude $1800 \mathrm{~m}$ a.s.l.; low, altitude $1600 \mathrm{~m}$ a.s.l. 
441 Table 4. Number of ASVs characteristic to low, medium and high altitudes and their taxonomic 442 affiliation.

\begin{tabular}{|c|c|c|c|c|c|c|c|}
\hline \multirow[b]{2}{*}{ Phylum } & \multirow[b]{2}{*}{ Taxon } & \multicolumn{2}{|l|}{$\underline{\text { Low }}$} & \multirow{2}{*}{$\frac{\text { Medium }}{\text { Enriched }}$} & \multicolumn{3}{|c|}{$\underline{\text { High }}$} \\
\hline & & Enriched & Depleted & & Depleted & Enriched & Depleted \\
\hline \multirow[t]{11}{*}{ Acidobacteria } & Bryobacter & & & & & 7 & 2 \\
\hline & Candidatus Solibacter & & & & & 6 & \\
\hline & Granulicella & & & & & 1 & \\
\hline & Occallatibacter & & & & & 1 & \\
\hline & unclassified Acidobacteriales & & & & & 1 & \\
\hline & uncultured Acidobacteriaceae (Subgroup 1) & & & & & 4 & \\
\hline & uncultured Acidobacteriales & 1 & & & & 16 & 1 \\
\hline & Pyrinomonadaceae clade RB41 & & & & & 3 & \\
\hline & Subgroup 7 & 1 & & & & 2 & 1 \\
\hline & Subgroup 6 & & & & & 4 & \\
\hline & Angustibacter & & & & & 1 & \\
\hline \multirow[t]{7}{*}{ Actinobacteria } & Terrabacter & & & & & 1 & \\
\hline & unclassified Kineosporiaceae & & & & & 1 & \\
\hline & uncultured Frankiales & 1 & & & & & 1 \\
\hline & Class MB & & & & & 1 & \\
\hline & Gaiella & & & & & 1 & \\
\hline & Patulibacter & & & & & & 1 \\
\hline & uncultured Gaiellales & & & & & 3 & \\
\hline Bacteroidetes & Flavisolibacter & & & & & 1 & 2 \\
\hline $\mathrm{AD} 3$ & Phylum AD3 & & & & & 2 & \\
\hline \multirow[t]{5}{*}{ Chloroflexi } & uncultured Roseiflexaceae & & & & & 1 & \\
\hline & Family JG30 & & & & & 3 & \\
\hline & Order B12 & & & & & 1 & \\
\hline & Order C0119 & & & & & 2 & \\
\hline & Cluster TK10 & & & & & 3 & \\
\hline \multirow[t]{4}{*}{ Gemmatimonadetes } & Gemmatimonas & 1 & & & & 7 & 3 \\
\hline & Gemmatirosa & & & & & 3 & \\
\hline & unclassified Gemmatimonadaceae & 2 & 1 & & & 4 & 3 \\
\hline & uncultured Gemmatimonadaceae & & & & & 11 & \\
\hline Nitrospirae & Nitrospira & & & & & 3 & \\
\hline Patescibacteria & family Saccharimonadales & & & & & 1 & 1 \\
\hline \multirow[t]{9}{*}{ Proteobacteria } & Bradyrhizobium & & & & & 1 & \\
\hline & Phenylobacterium & 1 & & & & 1 & 1 \\
\hline & Pseudolabrys & & & & & 1 & \\
\hline & Sphingobium & & & & & & 2 \\
\hline & Sphingomonas & & & & & 8 & 2 \\
\hline & unclassified Xanthobacteraceae & & & & & 2 & \\
\hline & uncultured Alphaproteobacteria & & & & & 1 & \\
\hline & uncultured Caulobacteraceae & & & & & 2 & \\
\hline & uncultured Elsteraceae & & & & & 2 & \\
\hline
\end{tabular}


uncultured Elsterales

uncultured Rhodospirillaceae

1

uncultured Xanthobacteraceae

Anaeromyxobacter

Haliangium

Pajaroellobacter

family A21b

Burkholderia

Caenimonas

order Ellin6067

2

Lysobacter

Massilia

1

1

MND1

2

Ramlibacter

Family SC

unclassified Methylophilaceae

5

1

4

1

3

1 


\section{Supplementary Files}

This is a list of supplementary files associated with this preprint. Click to download.

- SupplementaryFigS1.docx

- SupplementaryTables1.docx

- SupplementaryTableS2.docx 